\title{
Oral health, mouthwashes and cancer - what is the story?
}

\author{
Abstracted from \\ Guha N, Boffetta P, Wünsch Filho V, et al. \\ Oral health and risk of squamous cell carcinoma of the head and neck and esophagus: \\ results of two multicentric case-control studies. Am J Epidemiol 2007; 166: 1159-1173
}

Address for correspondence: Dr. Paul Brennan, Genetic Epidemiology Group, Genetics and Epidemiology Cluster, International Agency for Research on Cancer, 150 cours Albert Thomas, 69008 Lyon, France. E-mail: brennan@iarc.fr

\section{Question: What are the lifestyle, occupational and genetic risk factors for head and neck and oesophageal cancers?}

Design Two hospital-based case-control studies were conducted in central and eastern Europe and Latin America.

Case / control selection Cases and controls were recruited in Moscow (Russia), Bucharest (Romania) and Lodz and Warsaw (Poland) from 1998 to 2002, and from 1998 to 2003 in Buenos Aires (Argentina), La Habana (Cuba) and the Brazilian towns Rio de Janeiro, São Paulo, Pelotas, Porto Alegre and Goiânia. Incident cases of squamous cell carcinoma of the head and neck (oral cavity, pharynx, larynx) and oesophagus, as well as age- (in quinquennia) and sex frequencymatched controls, were enrolled from 1998 to 2003. Control subjects included residents of the study areas for at least 1 year who were admitted to the same hospitals as the cases or in a comparable catchment area (controls in São Paulo were not recruited from oncology hospitals, and population-based controls were enrolled in Warsaw). Controls were hospitalised for prespecified conditions thought to be unrelated to tobacco use or alcohol consumption. Both studies were coordinated by the International Agency for Research on Cancer according to an identical protocol for both case and control recruitment.

Data analysis Data from the central European and Latin American studies were analysed separately. Multivariable logistic regression models, including terms for oral health indicators, age, sex, education, country (central Europe) or centre (Latin America), tobacco pack-years and cumulative alcohol consumption, were used to obtain odds ratios (OR) and $95 \%$ confidence intervals $(\mathrm{Cl})$. All oral hygiene indicators and covariates were analysed as categorical variables, except for age, cumulative alcohol consumption, tobacco pack-years, and age when full-time education was completed, which were analysed as continuous variables. Case-control comparisons were made using $t$ tests for continuous variables and chi-square tests for categorical variables; two-sided $P$ values were obtained. Effect modification was assessed by stratifying on smoking (never/ former/ current) and drinking (never/ ever) habit. Heterogeneity between centres was tested.

Results Risk factors for head and neck cancer, independent of tobacco use and alcohol consumption, were as follows: poor condition of the mouth [central Europe OR, 2.89 (95\% Cl, 1.74-4.81); Latin America $\mathrm{OR}, 1.89$ (95\% Cl, 1.47-2.42)]; lack of toothbrush use [Latin America OR, $2.36(95 \% \mathrm{Cl}, 1.28-4.36)$ ], and daily mouthwash use [Latin America OR, 3.40 (95\% Cl,1.96-5.89]. Missing six to 15 teeth was an independent risk factor for oesophageal cancer [central Europe
OR, 2.84 (95\% Cl, 1.26-6.41); Latin America OR, 2.18 (95\% Cl, 1.04-4.59)].

Conclusions These results indicate that periodontal disease (as indicated by poor condition of the mouth and missing teeth) and daily mouthwash use may be independent causes of cancers of the head, neck and oesophagus.

\section{Commentary}

The UK newspapers have recently been full of headlines linking mouthwash to oral cancer, with varying degrees of certainty reported, for example:

"Mouthwash 'causes oral cancer' and should be pulled from supermarkets, say experts" (from the Daily Mail, 13 January 2009; see www.dailymail.co.uk/health/article-1113422/Mouthwash-causesoral-cancer-pulled-supermarkets-say-experts.html)

"Mouthwash linked to cancer" (Daily Telegraph, 11 January 2009; see www.news.com.au/dailytelegraph/story/0,22049,248965835001021,00.html)

"Mouthwash can raise cancer risk" [3] (Metro, 12 January 2009; see www.metro.co.uk/news/article.html?Mouthwash_can_raise_ cancer_risk\&in_article_id=474348\&in_page_id=34).

All of these articles have picked up on a recent narrative review of the literature published in Australian Dental Journal, ${ }^{1}$ whose authors present their own subjective opinion about the evidence for the risks associated with alcohol-based mouthwash. Their review did include - although did not fully represent - the significant research work from the Genetic Epidemiology Group, Genetics and Epidemiology Cluster, International Agency for Research on Cancer (IARC). 2 IARC have recently completed a high quality major international multicentre case-control study - including centres from across Europe, Russia, Latin and South America. This study is a concerted pooling effort to obtain substantial numbers of head and neck cancer cases, namely 924 cases and 928 controls in Europe and 2,286 cases and 1,824 controls in Latin America. The study goes much further than mouthwash use, examining a range of oral health and oral hygiene behaviours in relation to head and neck cancer risk.

The key findings are important for the dental profession and for informing public health approaches. For the European data they are:

- poor oral health is associated with an almost threefold increased risk of head and neck cancer;

- but missing teeth; lack of toothbrushing; wearing a denture were all not associated with an increased risk of head and neck cancer in Europe. The European study did not assess mouthwash use; gum 
bleeding; regular or irregular dental check-ups; using other oral hygiene instruments or using toothpaste. The Australian mouthwash review therefore quotes selectively only the Latin American data in relation to mouthwash - in which a three-fold increased risk was found.

So where does that leave us? Certainly the data are open to debate and further investigation is needed. Lessons around the presentation of data need to be taken on board to avoid the sensational scare mongering headlines. This is particularly important for the way risk is reported and interpreted. There is also little doubt that the role of oral health and oral health behaviours in the aetiology of head and neck cancer has not received the attention it deserves. This is especially of concern given the anatomical proximity and common risk factors associated with both cancer of the head and neck cancer and other oral conditions. Perhaps the reason for this is the accepted mantra that head and neck cancers are only caused by smoking and alco- hol consumption. This has been to the detriment of considering other aetiological factors in research.

The role of oral health warrants further investigation and this could include a systematic review and meta-analysis of the world literature and, potentially and ideally, a pooled analysis of the individual patient data. I understand that the International Head and Neck Cancer Epidemiology consortium have this research on their agenda.

\section{David Conway}

Dental Public Health Unit, Dental School, Medical Faculty, University of Glasgow, Glasgow, Scotland, UK

1. McCullough MJ, Farah CS. The role of alcohol in oral carcinogenesis with particular reference to alcohol-containing mouthwashes. Aust Dent / 2009; 53: 302-305.

2. Guha N, Boffetta P, Wunsch Filho V, et al. Oral health and risk of squamous cell carcinoma of the head and neck and esophagus: results of two multicentric casecontrol studies. Am J Epidemiol 2007; 166: 1159-1173.

Evidence-Based Dentistry (2009) 10, 6-7. doi:10.1038/sj.ebd.6400624 